\title{
Biotechnological Potential of Mushrooms: Drugs and Dye Production
}

\author{
Mahendra Rai \& Girish Tidke \\ Department of Biotechnology, Amravati Univarsity, Amravati, Maharashtra - 444602, Iindia
}

Mushrooms are higher basidiomycetous fungi of immense biotechnological importance. Most mushrooms represent an unlimited source of secondary metabolites of high medicinal value. Since ancient times, mushrooms have been valued as an edible and medicinal resource by tribal and local people of India. The large numbers of biologically active molecules have been identified in many mushroom species in India and all over the world. The search for new medicinal antibiotic substances from higher Basiodimycetes and the study of the medicinal value of these wild edible and medicinal mushrooms has become a matter of great interest in India. These naturally occurring biologically active antibiotic substances can be obtained from the mushrooms.

There is a wide range of bioactivity in mushrooms, which include antitumor activity of polysaccharides; antimicrobial and antiparasitic effects; antibiotics; antidiabetic, anti-hyperlipidemic, and antiaging benefits. They also can be used in the treatment of hepatitis B. We evaluated 17 mushrooms for antimicrobial activity. Most of them belonged to the group Polyporales, which showed remarkable activity against human pathogenic bacteria and fungi. The tests were carried out with hot water extracts of the mushrooms. The disc diffusion technique was used for the detection of activity. Results indicated that these mushrooms could be used for the preparation of antimicrobial agents.

Natural and ecofriendly organic dyes are very useful as coloring agents. These dyes are found in naturally collected or artificially growing fruit bodies, pure culture mycelia, and culture filtrate of mushrooms. There is now renewed demand for natural organic dyes from national and international markets because they provide better quality dyes for food, medicines, and cosmetics. The textile industry has also accepted natural dyes because they produce permanent color without damaging the fiber, thereby yielding more profitable product. These dyes are also used for making paints. And the remains of mushroom are used to make paper.

We have screened mushrooms from Amravati and its suburbs for dye production. The main aim of the present study was to screen the mushrooms of the Amravati region for antimicrobial activity against human pathogenic bacteria and fungi and also for dye production, which can be used as natural and ecofriendly dyes. 